\title{
Using digital technology to help identify children with brain based visual issues
}

\author{
Nicola McDowell \\ n.mcdowell@massey.ac.nz \\ Massey University, New Zealand
}

Keywords: Brain based/cerebral visual impairment, iPad App, eye tracking, screening technology

\begin{abstract}
Brain based, or cerebral visual impairments (CVI) are the most common cause of vision impairment affecting children in the economically developed world, with a prevalence rate of $3.4 \%$ of children in mainstream education (Williams et al., 2021). However, many of these children are going unrecognized or misdiagnosed due to the challenges of identifying the visual issues commonly associated with CVI. CVI has recently been defined as a verifiable visual dysfunction which cannot be attributed to disorders of the anterior visual pathways of any potentially co-occurring ocular impairment (Sakki et al., 2018). CVI can affect the basic visual functions, such as visual acuity, visual fields and contrast sensitivity and also, the higher visual functions or visual perceptual abilities. Currently, children with issues with their basic visual functions are more often being identified as having CVI. Whereas, children with issues with their higher visual functions are often going undiagnosed, due to having normal or near normal visual acuity (Chandna et al., 2021).
\end{abstract}

This has highlighted the need for a novel, yet simple way to screen for higher visual function issues in children. As part of her doctoral research, the author developed the Austin Assessment for this purpose. Following the success of the initial research, which used playing cards in real time, the Austin Assessment was made into an App for iPads. The Austin Assessment is a simple activity of matching cards over five levels, with each level increasing the number of cards and pairs. The shapes on the cards on the App have been developed to for intrinsic crowding and visual crowding as the numbers of cards is increased. This allows the App to measure the key features of higher visual function issues, including: random search pattern using darting eye movements, increased search times (especially as the complexity increases) and decreases in performance when the numbers of objects increases (Bennett et al., 2018; Zihl \& Dutton, 2015).

The Austin Assessment App has been developed for the latest iPad with the TrueDepth camera, as this allows the child's eyes to be tracked as they are completing the assessment and provides real time data on eye movement in the results section of the App. The App also measures time taken and accuracy in matching the pairs, as the initial research showed that children with higher visual function issues took twice as long as children without visual issues to match the pairs and also, that they were less accurate.

In terms of helping to identify children with CVI, the App has the potential to be used as a screening tool to identify any children that warrant further assessment. Research is currently being undertaken to validate the Austin Assessment and also, to create a database of normative ranges by age group. Although developed as an assessment, the Austin Assessment can also be used as a training tool to improve visual abilities and different themes will be included in the App in future versions.

\section{References}

Bennett, C. R., Bailin, E. S., Gottlieb, T. K., Bauer, C. M., Bex, P. J., \& Merabet, L. B. (2018). Virtual Reality Based Assessment of Static Object Visual Search in Ocular Compared to Cerebral Visual Impairment. In Antona M. \& S. C. (Eds.), Universal Access in Human-Computer Interaction. Virtual, Augmented, and Intelligent Environments (Vol. 10908, pp. 28-38). Springer. https://doi.org/10.1007/978-3-319$\underline{92052-8 \quad 3}$ 


\section{SCHOLARSHIP OF TECHNOLOGY ENHANCED LEARNING}

SoTEL

\section{SoTEL Symposium 16-18 February 2022}

Chandna, A., Ghahghaei, S., Foster, S., \& Kumar, R. (2021). Higher Visual Function Deficits in Children With Cerebral Visual Impairment and Good Visual Acuity. Front Hum Neurosci, 15, 711873. https://doi.org/10.3389/fnhum.2021.711873

Sakki, H. E. A., Dale, N. J., Sargent, J., Perez-Roche, T., \& Bowman, R. (2018, Apr). Is there consensus in defining childhood cerebral visual impairment? A systematic review of terminology and definitions. British Journal of Ophthalmology, 102(4), 424-432. https://doi.org/10.1136/bjophthalmol-2017-310694

Williams, C., Pease, A., Warnes, P., Harrison, S., Pilon, F., Hyvarinen, L., West, S., Self, J., Ferris, J., \& Group, C. V. I. P. S. (2021, Jun). Cerebral visual impairment-related vision problems in primary school children: a cross-sectional survey. Dev Med Child Neurol, 63(6), 683-689. https://doi.org/10.1111/dmcn.14819

Zihl, J., \& Dutton, G. N. (2015). Cerebral Visual Impairment in Children. Springer. https://doi.org/10.1007/978$\underline{3-7091-1815-3}$ 